\title{
Internal Stirring: An Approach to Approximate Evaluation of Shielding Effectiveness of Small Slotted Enclosures
}

\author{
Andrzej Rusiecki ${ }^{1}$, Karol Aniserowicz ${ }^{2 *}$, Alistair P. Duffy ${ }^{3}$, and Antonio Orlandi ${ }^{4}$ \\ ${ }^{1}$ Plum Ltd., Wspolna 19, Ignatki, 16-001 Kleosin, Poland \\ ${ }^{2}$ Bialystok University of Technology, Wiejska 45D, 15-351 Bialystok, Poland \\ ${ }^{3}$ De Montfort University, The Gateway, Leicester, LE1 9BH, UK \\ ${ }^{4}$ University of L’Aquila, UAq EMC Laboratory, I-67100, L’Aquila, Italy \\ *k.aniserowicz@pb.edu.pl
}

\begin{abstract}
A new technique for approximate evaluation of a small slotted enclosure shielding effectiveness (SE) is proposed. The enclosure under test is equipped with a built-in, rotating, conductive stirrer, and the SE is determined for discrete angles of the stirrer position. The SE characteristics computed in the frequency domain for the enclosure with the stirrer are compared to those of the enclosure containing different arrangements of printed circuit boards. The paper concludes that the inclusion of an internal stirrer is a potentially beneficial approach to shielding effectiveness testing.
\end{abstract}

\section{Introduction}

Electromagnetic shielding phenomena are of substantial interest in the EMC and signal integrity domains and many aspects have been widely studied as discussed below. For practical enclosures, shielding effectiveness (SE) must be determined using meticulous measurements or computations.

The SE for different materials including perforated sheets has been calculated analytically, as in [1]. The guidelines presented in that work can also be adapted to estimate the shielding performance of multislot enclosures.

SE measurements of relatively large structures, such as shielded rooms, have been standardized [2]. Small enclosures can be examined on standard test sites, as in [3]-[10], however, the existing standards are not concerned with methods of testing of small devices. SE measurements and calculations are typically based on comparisons of field intensity in both the presence and absence of the tested enclosure.

Analytical models to calculate SE as a function of frequency for simple enclosures are described, for example, in [11]-[15]. The impact of slots, holes, and internal packaging on the shielding performance of the enclosure was investigated in [12]-[21]. The impact of filling the enclosure with simple metallic or lossy objects on SE was considered in [16]-[21]. The shielding features are also dependent on the location of a signal source or a field probe, inserted into the enclosure [11]-[21]. Differences between SE of electric and magnetic fields of large wire grid shields and effects of changing of the grid density were studied in [22]-[23]

A fundamental difference between the larger enclosures and the small enclosures investigated here, is that the smaller enclosures usually have a much greater 'fill factor' as they are likely to be relatively full 
of electronic components. However, the shielding characteristics of small enclosures filled with objects that mimic a realistic internal setup are under-researched.

In industrial practice, one often wishes to purchase a ready-to-use enclosure for an application that requires a specific minimum level of SE within a certain frequency band. Manufacturers provide charts specifying SE for empty enclosures only. This data may be used only as a starting point for the estimation of the shielding performance, since SE characteristics varies considerably depending on the enclosure filling. Hence, it would be of value if the manufacturers could include in their product specifications the SE characteristics determined for specific internal arrangements.

In this work we present a technique, which makes it possible to perform the worst case approximation to the SE of the enclosure filled with different configurations of electronic devices. The desired filling is simulated by inserting metal stirrers. Perturbation of the electromagnetic field distribution by the use of metallic stirrers is common for reverberation chamber measurements [24]-[26]. The proposed technique, with the use of conductive stirrers inside the enclosure under test, seems to be somewhat similar to the measurements in the nested reverberation chamber setup, but the stirrer's role is different. For example, Holloway et al. in [9] analyse the use of the nested reverberation chamber and frequency stirring, which may be considered as an alternative technique to that presented here, but they did not study the possibility of modelling of different internal loading of the enclosure. Gifuni in [27] examines the relation between the enclosure SE and the wall material. Wang et al. in [10] present a technique of measurements of average and minimum SE basing on a multipoint sampling along an axis of a tested enclosure, however, only an empty enclosure is considered.

The stirrers applied here occupy a substantial part of the enclosure volume and they are used to simulate different internal setups that cause changes of the internal field distribution and thus the enclosure shielding performance. The use of the internal stirrers to simulate a number of the enclosure internal setups has an advantage of performing the worst case SE evaluation in a single cycle of measurements. The number of operations concerning the reconfiguration of the internal arrangement as well as changing the placement of the signal source inside the enclosure can be reduced thus minimizing the time necessary for the experiments. The measurements described in this work were carried out in a GTEM cell [28]-[30], but they may be performed using other test sites, as well.

The concept of internal stirring has already been presented in [31]-[33]. In the present work a detailed analysis of the impact of the internal stirrer position on the SE is provided. Different locations of a monopole antenna inside the enclosure are considered. FEKO suite [34], based on the Method of Moments (MoM), was used for computations in [31]-[33]. Here, COMSOL [35] is applied, which is a Finite 
Element Method (FEM) code whose numerical technique is intrinsically different from MoM. Furthermore, a cross validation of the numerical models is performed by comparing the FEKO results versus those obtained by using COMSOL and the measured ones.

\section{Validation of measurements and computations}

For the sake of the comparison of results, an empty enclosure was selected that has been investigated by many researchers [12]-[20]. It is a metallic box of dimensions $300 \times 300 \times 120 \mathrm{~mm}$ with $100 \times 5 \mathrm{~mm}$ aperture in one of its walls (Fig. 1a). The coordinate system origin is located in the centre of the box.

A physical model of the enclosure was made of $0.32 \mathrm{~mm}$ tin plated steel sheets soldered together. A 60-mm monopole antenna of 2-mm diameter was inserted into the box. The monopole was fixed directly to the centre pin of the $\mathrm{N}$-type connector on the box top side (Fig. 1a). A $50 \Omega$ coaxial cable joined the connector with the measuring equipment (Fig. 2).

The study was carried out doing both computation analysis and experimentation, which were performed in absence and in presence of the steel enclosure. The former configuration is the reference one. The reference values were determined using the structure composed by the monopole and its reference plane (Fig 1b). All the remaining walls were removed from the reference model.
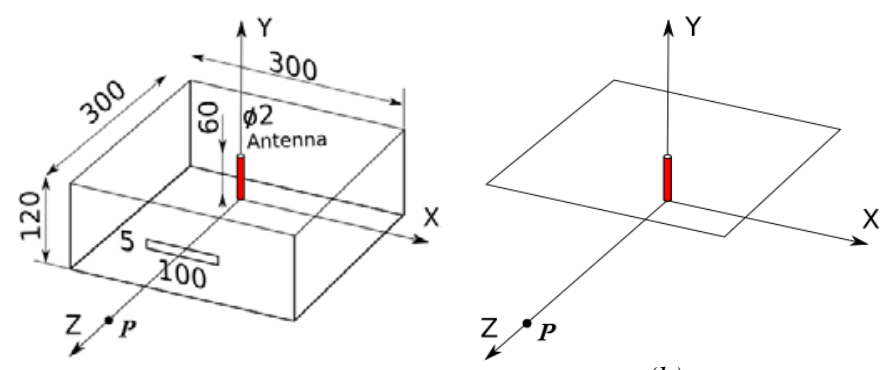

(a)

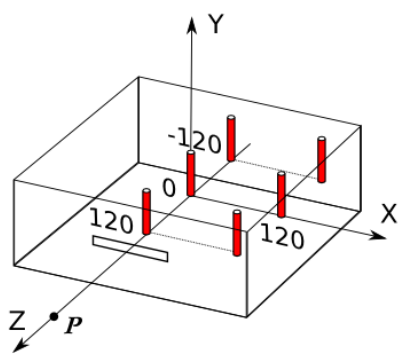

(b)

Fig. 1. Analysed enclosure (not to scale)

(c)

a Dimensions in $\mathrm{mm}$

b Reference plane

c Locations of the antenna monopole

The experiments were carried out in the Schaffner GTEM 1500 cell (Fig. 2). The measurement setup consisted of the Rohde \& Schwarz FSH3 vector analyzer equipped with additional amplifier and 
attenuator. The attenuator was used to protect the power amplifier input. The used equipment can work in the frequency band from $80 \mathrm{MHz}$ to $3 \mathrm{GHz}$. The tested device was placed directly on the GTEM cell floor to avoid the influence of a parasitic capacitance between the enclosure and the floor. This capacitance forms a resonant circuit in combination with the coaxial cable, which can have significant impact on the measurement results.

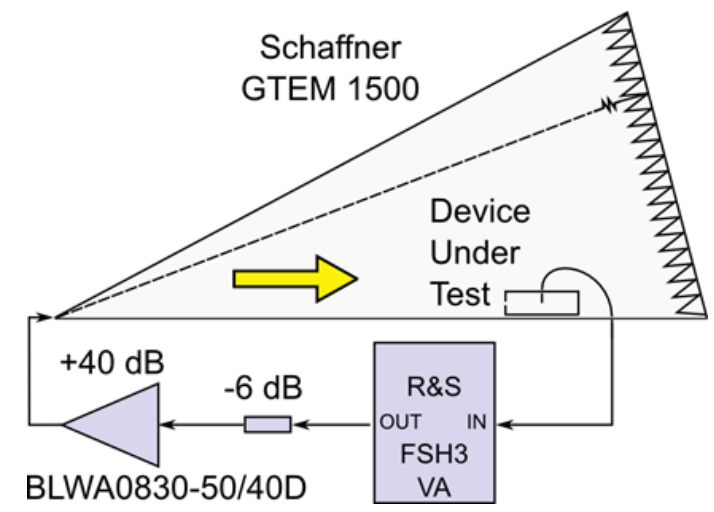

Fig. 2. Measurement setup with GTEM cell

The tested enclosure was illuminated by an electromagnetic wave from the direction perpendicular to the slotted wall. The SE was obtained by comparison of two sets of results: the reference values with the enclosure absent (Fig. 1b), and the values affected by the enclosure present (Fig. 1a).

In [31]-[33] the FEKO suite [34] was used for analysis of a similar problem. The model of the enclosure was illuminated by the electromagnetic plane wave. The enclosure wall thickness was taken into account. Here, the COMSOL computer code [35], employing the Finite Element Method, is applied for comparison of results of different numerical approaches. The computations become very time consuming if the numerical model is so large that the disk virtual memory has to be used. For the sake of minimization of the number of finite elements, the walls of the enclosure are assumed to be infinitely thin. The model is perfectly conducting. The enclosure is surrounded by a sphere of radius $400 \mathrm{~mm}$ (Fig. 3), with absorbing boundary conditions. While using COMSOL, the direction of the electromagnetic radiation is reversed: the antenna monopole contains an excitation source. The antenna model is divided into six 1cm segments, and the source segment touches the box top side. The SE is computed at chosen point $P$ as

$$
\mathrm{SE}_{\mathrm{E}}(\omega, P)[\mathrm{dB}]=20 \log \frac{\left|E_{0}(\omega, P)\right|}{\left|E_{S}(\omega, P)\right|},
$$

where $E_{0}(\omega, P)$ is the reference value computed in the enclosure (shield) absence, and $E_{S}(\omega, P)$ is computed in the enclosure presence. The equivalence of results obtained for both directions of propagation was experimentally confirmed in [33]. 


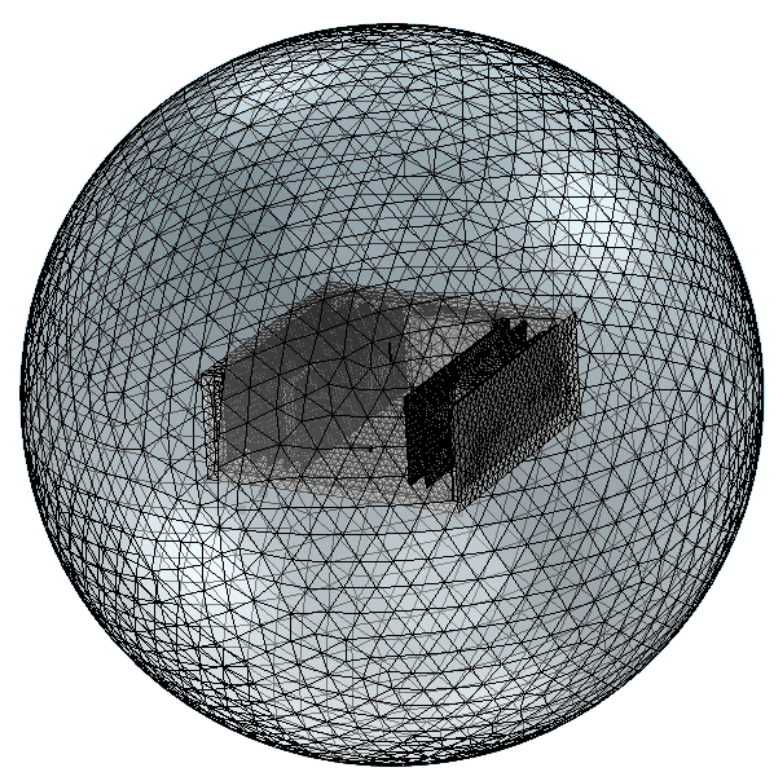

Fig. 3. Finite element mesh (COMSOL)

Point $P$ is located outside the enclosure, at the $z$ axis (Fig. 1). The large number of elements used can cause difficulties in selecting the distance $r$ of point $P$ sufficiently far from the radiating source. The application of commonly used formulas for the far zone in a wide frequency band $\left(r_{\mathrm{far}}>>\lambda /(2 \pi)\right.$, $r_{\text {far }} \geq 2 D^{2} / \lambda$, where $D$ is the slot length, $\lambda$ - the wavelength) leads to an unacceptable increase of the analysed volume. To avoid unnecessary resource consumption, point $P$ was obtained by gradually increasing the distance between the slot and point $P$, and observing changes of plots of the electric $\operatorname{SE}_{\mathrm{E}}(\omega, P)$ and magnetic $\mathrm{SE}_{\mathrm{H}}(\omega, P)$ shielding effectiveness (from now on the indication of point $P$ will be dropped for the sake of brevity), so as to obtain acceptably small differences between those plots. In the far zone these curves are identical. Finally, a decision was made to locate point $P$ at a distance of $200 \mathrm{~mm}$ from the slot (coordinates: $x=0, y=0, z=350 \mathrm{~mm}) . \mathrm{SE}_{\mathrm{H}}(\omega, P)$ was computed using formula analogous to (1). Plots of $\mathrm{SE}_{\mathrm{E}}(\omega)$ and $\mathrm{SE}_{\mathrm{H}}(\omega)$ at the chosen point are presented in Fig. 4a. All the characteristics presented here (except for the one in Fig. 4a) were computed as electric $\mathrm{SE}_{\mathrm{E}}(\omega)$, according to formula (1). Index ' $E$ ' will be omitted.

As shown in Fig. 1c, six different locations of the monopole antenna inside the enclosure were considered in order to find the location assuring the strongest possible transmission through the aperture. The antenna was parallel to the $y$ axis because this orientation corresponds with the polarization of the electric field radiated from the slot. The $\mathrm{SE}(\omega)$ characteristics for the analyzed locations are shown in Fig. 4b. In the frequency range below $1 \mathrm{GHz}$, which is the most interesting in this case (for studies of the worst shielding performance), the strongest radiation from the aperture (the lowest SE curve, shown in red) is 
observed for the location of coordinates: $x=0, z=0 \mathrm{~mm}$ (see Fig. 1). Only this excitation point is considered further.

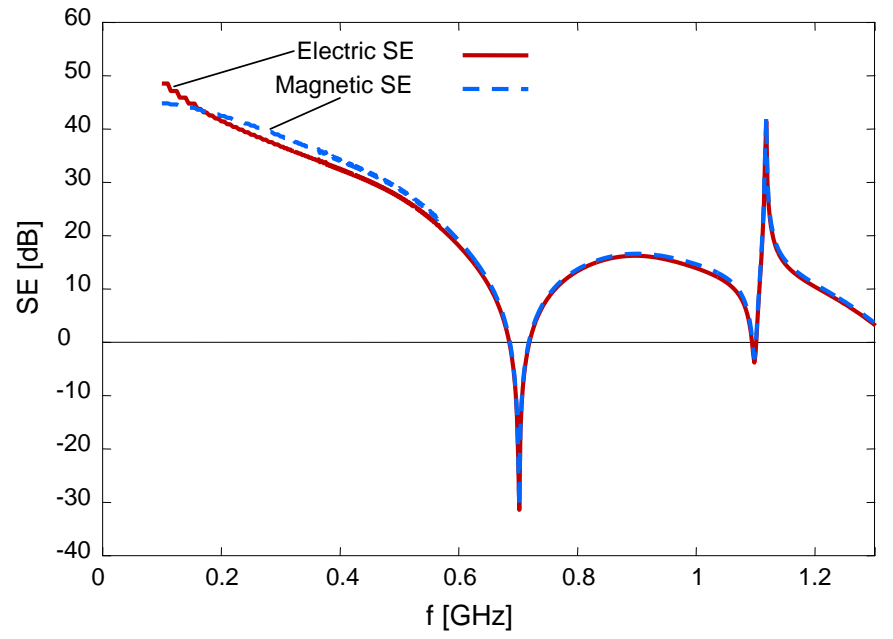

(a)

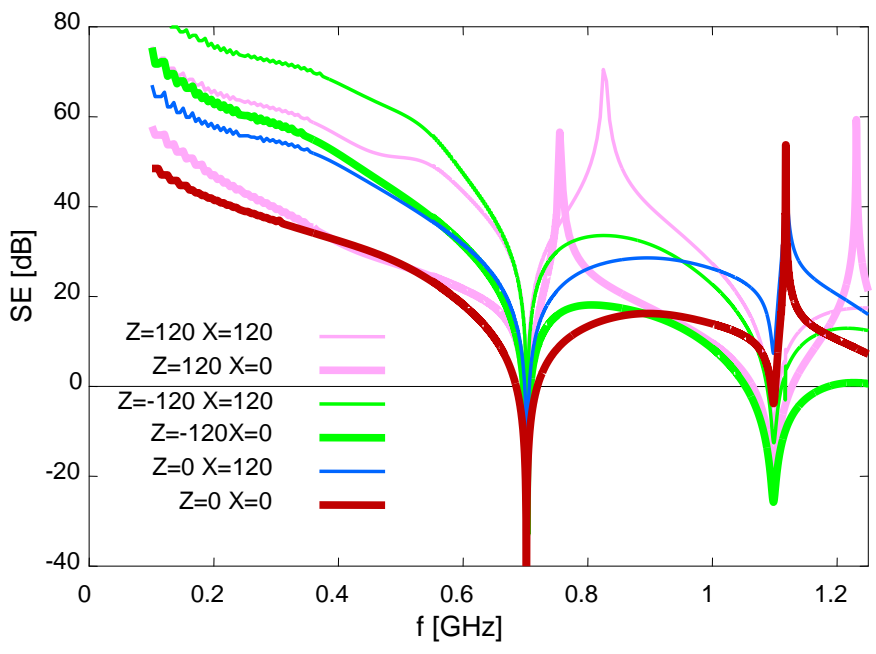

(b)

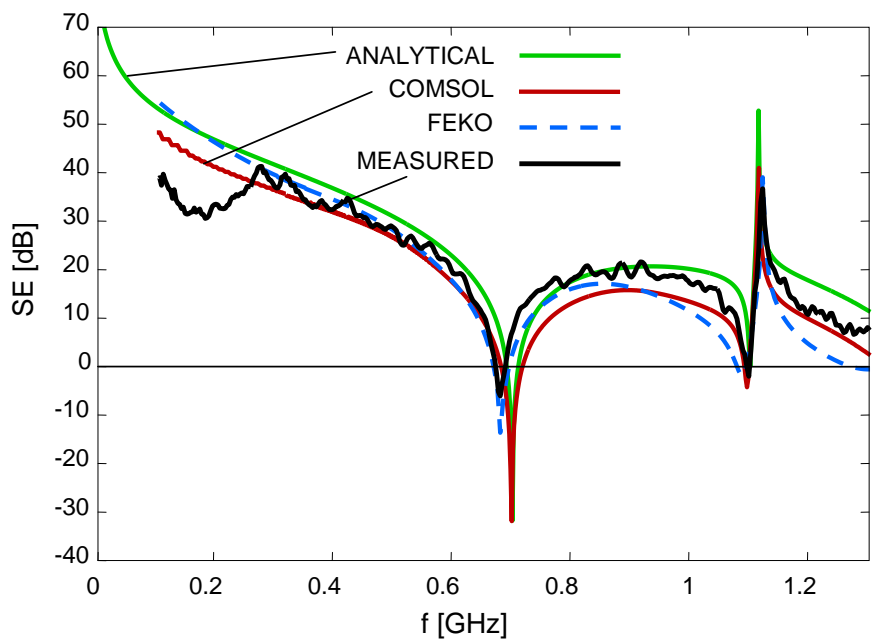

(c)

Fig. 4. Shielding effectiveness of empty enclosure

a Electric $\mathrm{SE}_{\mathrm{E}}(\omega)$ and magnetic $\mathrm{SE}_{\mathrm{H}}(\omega)$ computed at point $P$

b $\operatorname{SE}(\omega)$ computed for different locations of the antenna monopole

c Computed (COMSOL, FEKO), measured, and analytically calculated curves of $\operatorname{SE}(\omega)$

In the analyzed frequency range, the shielding enclosure is inefficient around $700 \mathrm{MHz}$ and 1100 $\mathrm{MHz}$, where $\mathrm{SE}<0$ regardless of the source location.

The calculated and measured characteristics of $\mathrm{SE}(\omega)$ are compared in Fig. 4c. The plot of the analytical expression is also presented. The analytical approach was published in [12] and [16] basing on representation of the enclosure by its equivalent circuit. All the curves, except for the COMSOL one, are determined for the direction of propagation shown in Fig. 2. Calculations using both COMSOL and FEKO 
for one frequency sample took about 25 seconds on a PC with quad-core processor Intel Core i7-3770K, 3.5 GHz and 32 GB of RAM. The time of computations using analytical formulas was negligible.

Both measured and computed results are in reasonably good agreement with each other and with those published in the literature [12]-[20]. The shift of the resonant frequency, similar to that presented in Fig. 4c, was noted also in [16]. There are also noticeable differences between the numerical and experimental results. The good agreement between experiments and FEKO for the lowest resonant frequency was obtained probably because of taking the wall thickness into account during computations. The COMSOL curve was obtained assuming the infinitely thin walls. The measurements at low frequencies, below $300 \mathrm{MHz}$, were affected by significant errors (Fig. 4c) because of limitations of the dynamic range of the measuring equipment available during experiments. Similar issues are found in the literature, e.g. [4], [5], [20]. Numerical instability was observed using COMSOL below $100 \mathrm{MHz}$, which can also be seen above $100 \mathrm{MHz}$ as slight rippling of the displayed curves. For this reason, only analytical results are presented for frequency approaching zero.

In order to quantify the level of agreement between the comparisons, in accordance with IEEE Standard P1597 [36], the Feature Selective Validation Technique (FSV) [37], [38] has been applied to the comparison of different pairs of datasets in Fig. 4c. Three total FSV measures are calculated: GDMtot the global difference measure, ADMtot - the amplitude difference measure, and FDMtot - the feature difference measure. Their numeric values have, attributed to them, a qualitative descriptor of the data agreement of 'Excellent', 'Very Good', 'Good', 'Fair', 'Poor' and 'Very Poor'. The lower the numeric value of $\mathrm{xDM}$ the better is the agreement of the datasets. The results are taken from the frequency range $300 \mathrm{MHz} \leq f \leq 1250 \mathrm{MHz}$ and they are summarized in Table I. As it is seen, the calculated FSV measures have been classified as 'Good' or 'Fair' (xDM values within the ranges of 0.2 and 0.4 , or 0.4 and 0.8 , respectively).

Table 1 FSV difference measures for data in Fig. 4c (from $300 \mathrm{MHz}$ to $1250 \mathrm{MHz}$ )

\begin{tabular}{lcccccc}
\hline & \multicolumn{2}{c}{ GDMtot } & \multicolumn{2}{c}{ ADMtot } & \multicolumn{2}{c}{ FDMtot } \\
\hline Measured vs. FEKO & 0.42 & Fair & 0.30 & Good & 0.28 & Good \\
\hline Measured vs. COMSOL & 0.55 & Fair & 0.31 & Good & 0.40 & Good \\
\hline Measured vs. analytical & 0.57 & Fair & 0.35 & Good & 0.46 & Fair \\
\hline COMSOL vs. FEKO & 0.60 & Fair & 0.36 & Good & 0.41 & Fair \\
\hline
\end{tabular}

Further analysis is presented in the frequency range up to $1250 \mathrm{MHz}$, although the investigations were performed also outside that band. It was observed that, at higher frequencies, the tested enclosure cannot be treated as an efficient shield. At higher frequencies the number of resonances increases and the 
resonant frequencies move along the abscissa axis depending on the enclosure internal arrangement (see Figs. 6 and 8).

\section{Influence of internal arrangement on shielding effectiveness}

Consider three different internal setups. They are presented in Fig. 5, and called PCB1, PCB2, and PCB3. The equipment inside the box is modelled as cards made of pure FR4 glass epoxy laminate, and with copper or aluminium elements of different shapes. The FR4 epoxy cards are of dimensions $250 \times 100$ $\times 2 \mathrm{~mm}$, and they are placed with $20 \mathrm{~mm}$ spacing. The FR4 material constants are: $\sigma=0,004 \mathrm{~S} / \mathrm{m}, \varepsilon_{r}=4.5$. The PCB1 and PCB2 setups are equipped with the FR4 base boards of $280 \times 280 \times 2 \mathrm{~mm}$. In the PCB1 setup, a large Cu block is a model of a shielded copper box with dimensions of $250 \times 100 \times 70 \mathrm{~mm}$. Small conductive elements are modelled as $4 \mathrm{~mm}$ or $3 \mathrm{~mm}$ thick solid copper cuboids with sizes of $100 \times 100$, $80 \times 20$, $50 \times 50$, or $25 \times 25 \mathrm{~mm}$, respectively. In the PCB2 setup, the copper elements mounted on additional two FR4 cards are of $80 \times 80 \times 4 \mathrm{~mm}$ and $50 \times 50 \times 3 \mathrm{~mm}$, respectively. Solid Cu elements, used in the PCB3 setup, have dimensions of $100 \times 100 \times 30$ and $50 \times 30 \times 25 \mathrm{~mm}$, respectively. The side chassis cards dimensions are of $250 \times 100 \times 2 \mathrm{~mm}$. The remaining elements of PCB3 are assumed to be made of aluminium.

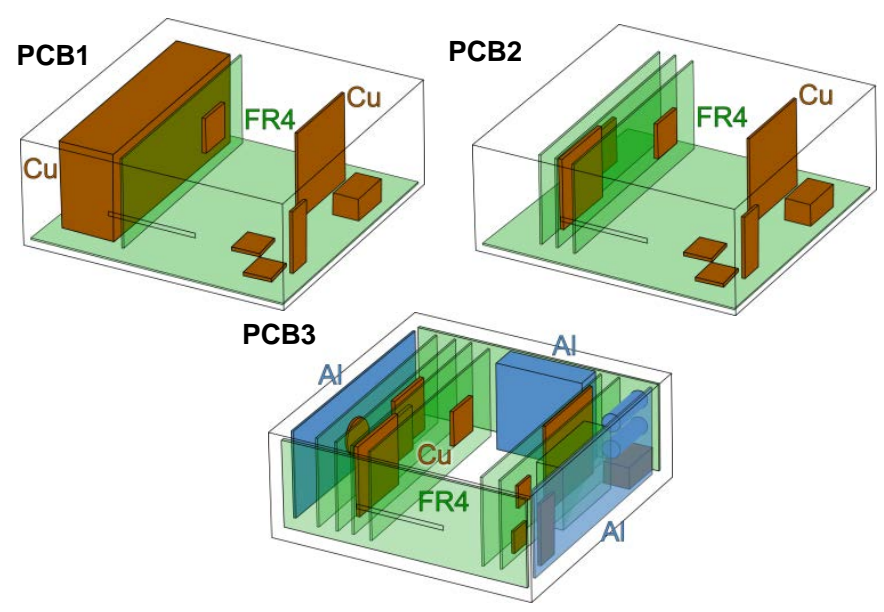

Fig. 5. Analysed internal arrangements

The finite element meshes generated by COMSOL code [35] consisted of 165778, 300816, and 514806 tetrahedral elements for PCB1, PCB2, and PCB3 arrangements, respectively. The corresponding numbers of degrees of freedom were 1060474, 1916172, and 3276776. The average CPU time per one frequency step was 38 s (PCB1), 91 s (PCB2), and 150 s (PCB3). 
The results are compared with the $\operatorname{SE}(\omega)$ of the empty enclosure in Fig. 6. The SE characteristics, including resonant frequencies, vary depending on the internal packaging of the enclosure.

The box may be used as an efficient shield in those frequency bands, in which $\operatorname{SE}(\omega)>0$. The resonances, around which $\mathrm{SE}(\omega) \leq 0$, cause the shield to become inefficient. For most practical applications, the frequency band below the first resonance is of interest. The internal filling of PCB1 causes that the upper limit of the useful low-frequency band moves from about $700 \mathrm{MHz}$ (for the empty box) down to about $490 \mathrm{MHz}$. The shielding properties of the enclosure are disputable in the range of $700 \mathrm{MHz}<f<950 \mathrm{MHz}$. For $f>950 \mathrm{MHz}$ the enclosure does not provide any protection against the electromagnetic emission for practical purposes. However, the use of this box as a shield is possible in a narrow band between $800 \mathrm{MHz}$ and $950 \mathrm{MHz}$, providing that a thorough investigation for a specific internal filling is carried out.

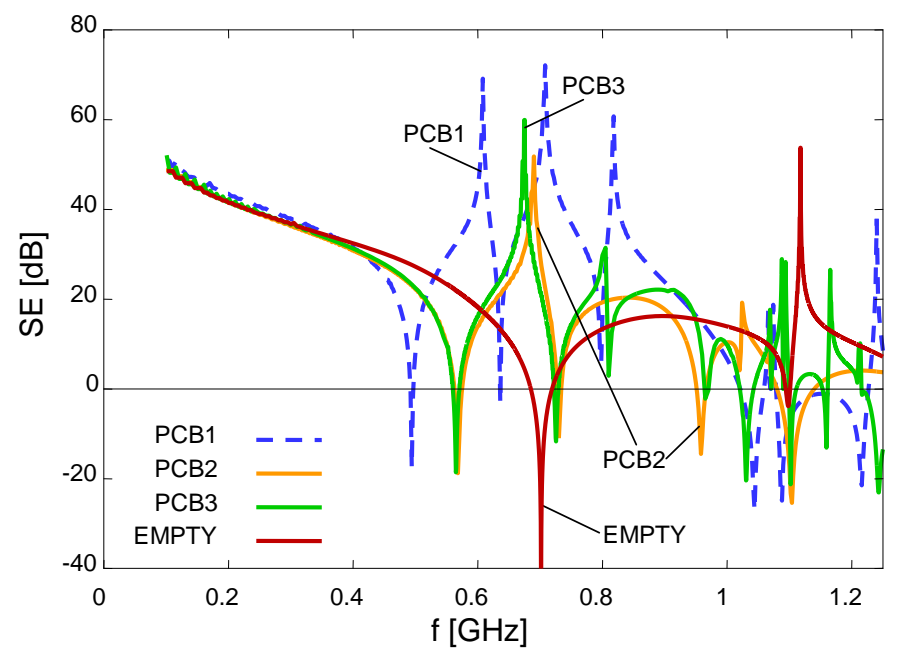

Fig. 6. $S E(\omega)$ of analyzed enclosure with different internal arrangements

The lower frequencies of the presented charts are of particular interest during the assessment of the enclosure shielding capability. The maximal resonant values of the $\operatorname{SE}(\omega)$ can be neglected from the shielding perspective. Note that resonant minimums are expected to reach values less than zero. Not each minimum observed in the presented charts is less than zero because of the finite frequency step of calculations ( $\Delta f=2 \mathrm{MHz}$ ) and to the present losses (due to both the radiation and the internal filling). 


\section{Shielding effectiveness of enclosure with internal stirrers}

Perfectly conducting stirrers are introduced to disturb the electromagnetic field distribution inside the analyzed empty box. The calculation results are presented here to prove that this concept may be applied as a method for modelling of the influence of varying internal filling on the shielding performance.

The influence of the stirrer's rotation on $\operatorname{SE}(\omega)$ is analyzed below. The coordinates of the stirrer's centre are: $x=75 \mathrm{~mm}, y=0 \mathrm{~mm}, z=0 \mathrm{~mm}$. Three stirrers of dimensions $100 \times 100 \times 0.32 \mathrm{~mm}$ and different axes of rotation are shown in Figs. 7 (a)-(c). These different cases are called STIR1a, STIR1b, and STIR1c, respectively.

Configuration STIR1c makes it possible to experiment with longer stirrers. Hence, a double-stirrer configuration called STIR2 was considered, of $200 \mathrm{~mm}$ in length (Fig. 7d). A configuration with a single long stirrer was examined in detail in [33] and it is not analysed here.

The $\operatorname{SE}(\omega)$ was calculated for discrete rotation angles of the stirrer, changing from 0 to 170 degrees, with the step of 10 degrees. The stirrer is symmetrical, so only halves of its full rotation are taken into account.
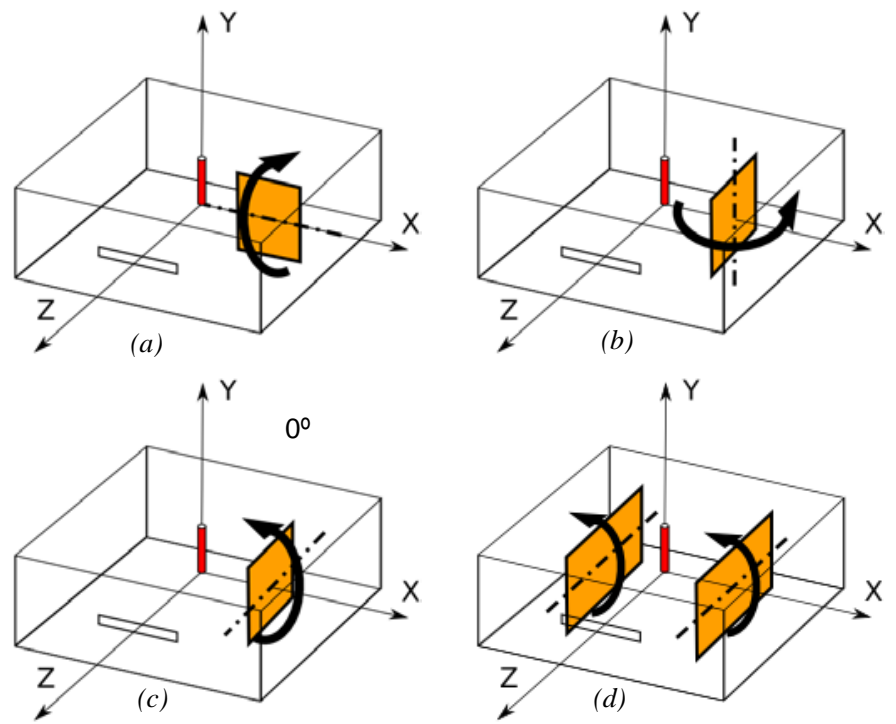

Fig. 7. Stirrers in different configurations:

a STIR1a

b STIR1b

c STIR1c

d STIR2

The SE $(\omega)$ characteristics for three configurations of stirrer STIR1 axis are presented in Figs. 8 (a)(c). It is seen that changes of the stirrer positions cause significant changes of $\operatorname{SE}(\omega)$. The smallest changes are observed for application of stirrer STIR1b, the largest - for STIR1c. Stirrer configuration STIR1c, 
which causes the largest dispersion of $\operatorname{SE}(\omega)$ resonances along the frequency axis, is the most effective for representation of possible changes of the enclosure fillings.

The stirrers in configuration STIR2 occupy much larger part of the analysed enclosure than that covered by STIR1. They are assumed to take synchronous turns in the odd and even directions. That stirrer set causes the largest dispersion of resonances among all the configurations analyzed here, so the SE characteristics for STIR2 (Fig. 8d) are used for further comparative studies.

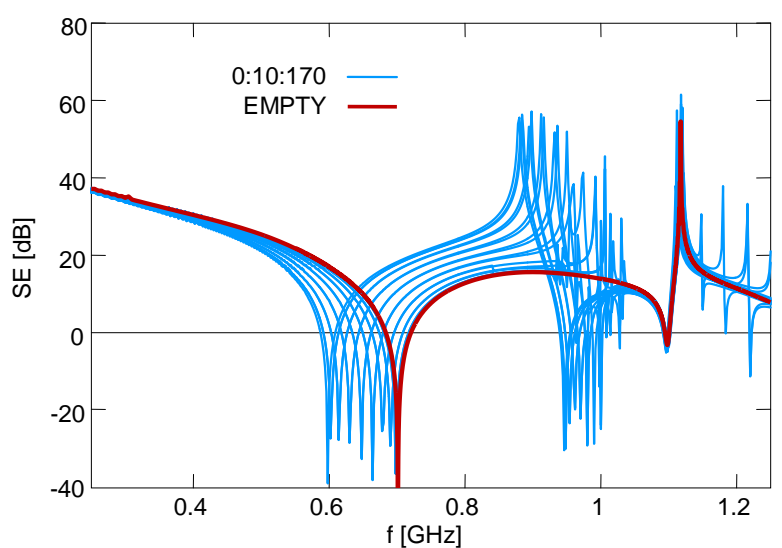

(a)

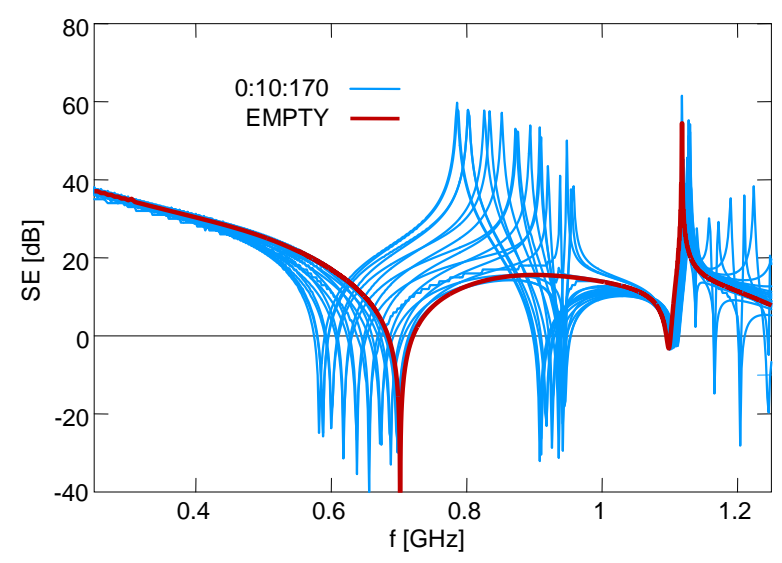

(c)

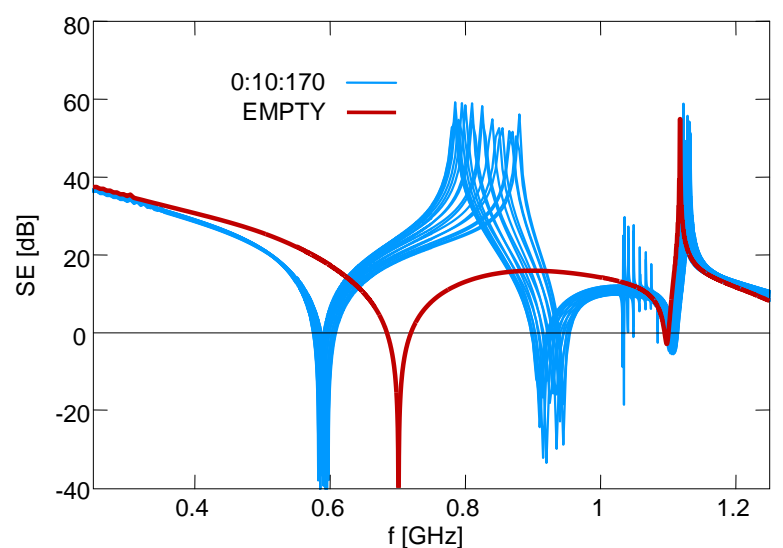

(b)

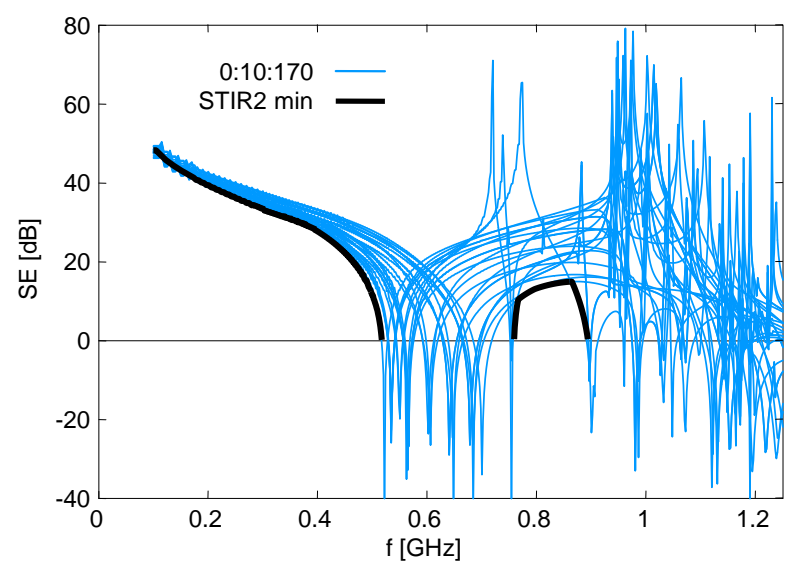

(d)

Fig. 8. $S E(\omega)$ of enclosure with stirrer:

a STIR1a

b STIR1b

c STIR1c

d STIR2 with its bottom envelope (STIR2 min)

\section{Evaluation of the worst shielding effectiveness}

The worst case of the enclosure shielding performance may be represented by the bottom envelope of the obtained SE curves, as in Fig. 8d. This worst case envelope is compared in Fig. 9 with the curves presented in Fig. 6. 
It is seen that the slotted enclosure under test can be used as an efficient shield in the frequency band below $500 \mathrm{MHz}$. In Fig. 8d, the band of $760 \mathrm{MHz}<f<890 \mathrm{MHz}$, where SE $>0$, should be treated with caution because of visible resonances found during the analysis of PCB1 and PCB3 arrangements (Fig. 9). It could be possible to modify the internal stirring in such a way that resonances are more dispersed than those presented in Fig. 8d. In our opinion however, the presented examples are sufficient to prove the effectiveness of the proposed internal stirring technique for the approximate evaluation of the enclosure worst shielding performance.

It should be expected that the enclosure manufacturer provides the customer with the worst case shielding characteristic, similar to the bottom envelope presented in Fig. 9. It is necessary to emphasize that this curve adds much information to the SE of the empty enclosure despite it is only the approximation of the worst case scenario. Additional investigations should be carried out if the customer wishes to know the SE for the actual internal arrangement, including the shielding performance in the higher frequency band.

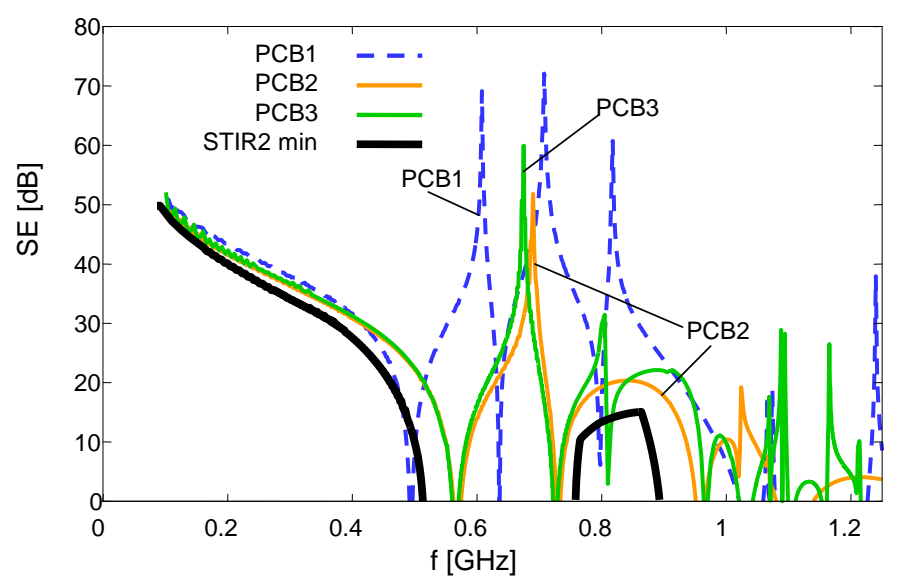

Fig.9. SE $(\omega)$ curves for different internal arrangements and bottom envelope of STIR2 characteristics (STIR2 min)

\section{Conclusion}

The technique of internal stirring described in this paper is a reliable approach for the approximate evaluation of the minimal SE of the tested enclosure, taking different internal fillings under consideration.

The selection of the best location of the antenna monopole results in the worst case of the SE characteristic.

The choice of the stirrer(s) is important for the correct evaluation of the minimal SE.

The internal stirring can be applied for both measurements and numerical analysis. The proposed technique is an efficient experimental tool owing to the fact that the measurements can be carried out in a 
single series of operations. However, if one wishes to obtain the actual SE characteristic, it is necessary to analyze the enclosure with its final internal arrangement.

\section{Acknowledgment}

This work was supported by Rector's Project S/WE/1/2015 of Bialystok University of Technology, Poland.

\section{References}

[1] Schulz R. B., Plantz V. C., and Brush D. R.: 'Shielding theory and practice', IEEE Trans. Electromagn. Compat., vol. 30, no. 3, Aug. 1988, pp. 187-201

[2] IEEE Std 299 ${ }^{\mathrm{TM}}$-2006: 'IEEE standard method for measuring the effectiveness of electromagnetic shielding enclosures’, Feb. 2007

[3] Fedeli D., Gradoni G., Primiani V. M., and Moglie F.: 'Accurate analysis of reverberation field penetration into an equipment-level enclosure’, IEEE Trans. Electromagn. Compat., vol. 51, no. 2, May 2009, pp. 170-180

[4] Dehkhoda P., Tavakoli A., and Moini R.: 'An efficient and reliable shielding effectiveness evaluation of a rectangular enclosure with numerous apertures’, IEEE Trans. Electromagn. Compat., vol. 50, no. 1, Feb. 2008, pp. 208-212

[5] Feng C., Shen Z.: 'A hybrid FD-MoM technique for predicting shielding effectiveness of metallic enclosures with apertures’, IEEE Trans. Electromagn. Compat., vol. 47, no. 3, Aug. 2005, pp. 456-462

[6] Gifuni A., Migliaccio M.: 'Use of nested reverberating chambers to measure shielding effectiveness of nonreciprocal samples taking into account multiple interactions’, IEEE Trans. Electromagn. Compat., vol. 50, no. 4, Nov. 2008, pp. 783-786

[7] Hatfield M. O.: 'Shielding effectiveness measurements using mode-stirred chambers: a comparison of two approaches', IEEE Trans. Electromagn. Compat., vol. 30, no. 3, Aug. 1988, pp. 229-238

[8] Holloway C. L., Hill D. A., Ladbury J., Koepke G., and Garzia R.: 'Shielding effectiveness measurements of materials using nested reverberation chambers’, IEEE Trans. Electromagn. Compat., vol. 45, no. 2, May 2003, pp. 350-356

[9] Holloway C. L., Hill D. A., Sandroni M., Ladbury J. M., Coder J., Koepke G., Marvin A. C., and He Y.: 'Use of reverberation chambers to determine the shielding effectiveness of physically small, electrically large enclosures and cavities', IEEE Trans. Electromagn. Compat., vol. 50, no. 4, Nov. 2008, pp. 770-782

[10] Wang Q., Cheng E., and Qu Z.: 'On the shielding effectiveness of small-dimension enclosures using a reverberation chamber’, IEEE Trans. Electromagn. Compat., vol. 53, no. 3, 2011, pp. 562-569

[11] Klinkenbusch L.: 'On the shielding effectiveness of enclosures', IEEE Trans. Electromagn. Compat., Aug. 2005, pp. 589-601

[12] Robinson M. P., Benson T. M., Christopoulos C., Dawson J. F., Ganley M. D., Marvin A. C., Porter S. J., and Thomas D. W. P.: 'Analytical formulation for the shielding effectiveness of enclosures with apertures', IEEE Trans. Electromagn. Compat., vol. 40, no. 3, Aug. 1998, pp. 240-248

[13] Solin J. R.: 'Formula for the field excited in a rectangular cavity with a small aperture', IEEE Trans. Electromagn. Compat., vol. 53, no. 1, Feb. 2011, pp. 82-90

[14] Wallyn W., De Zutter D., and Laermans E.: 'Fast shielding effectiveness prediction for realistic rectangular enclosures’, IEEE Trans. Electromagn. Compat., vol. 45, no. 4, Nov. 2003, pp. 639-643

[15] Liu Q.-F., Yin W.-Y., Mao J.-F., and Chen Z.: 'Accurate characterization of shielding effectiveness of metallic enclosures with thin wires and thin slots', IEEE Trans. Electromagn. Compat., vol. 51, no. 2, May 2009, pp. 293-300 
[16] Thomas D. W. P., Denton A. C., Konefal T., Benson T., Christopoulos C., Dawson J. F., Marvin A., Porter S. J., and Sewell P.: 'Model of the electromagnetic fields inside a cuboidal enclosure populated with conducting planes or printed circuit boards', IEEE Trans. Electromagn. Compat., vol. 43, no. 2, May 2001, pp. 161-169

[17] Araneo R., Lovat G.: 'Fast MoM analysis of the shielding effectiveness of rectangular enclosures with apertures, metal plates, and conducting objects', IEEE Trans. Electromagn. Compat., vol. 51, no. 2, May 2009, pp. 274-283

[18] Nie B.-L., Du P.-A., Yu Y.-T., and Shi Z.: 'Study of the shielding properties of enclosures with apertures at higher frequencies using the transmission-line modeling method', IEEE Trans. Electromagn. Compat., vol. 53, no. 1, Feb. 2011, pp. 73-81

[19] Shim J., Kam D. G., Kwon J. H., and Kim J.: 'Circuital modeling and measurement of shielding effectiveness against oblique incident plane wave on apertures in multiple sides of rectangular enclosure', IEEE Trans. Electromagn. Compat., vol. 52, no. 3, Aug. 2010, pp. 566-577

[20] Xue M., Yin W., Liu Q., and Mao J.: 'Wideband pulse responses of metallic rectangular multistage cascaded enclosures illuminated by an EMP', IEEE Trans. Electromagn. Compat., vol. 50, no. 4, Nov. 2008, pp. 928-939

[21] Primiani V. M., Moglie F., and Pastore A. P.: 'Field penetration through a wire mesh screen excited by a reverberation chamber field: FDTD analysis and experiments', IEEE Trans. Electromagn. Compat., vol. 51, no. 4, Nov. 2009, pp. 883-891

[22] Maksimowicz T., Aniserowicz K.: 'Simulation of currents induced in a loop by lightning strike of different waveforms', Przeglad Elektrotechniczny, vol. 86, no. 3, 2010, pp. 43-44

[23] Maksimowicz T., Aniserowicz K.: 'Investigation of models of grid-like shields subjected to lightning electromagnetic field: experiments in the frequency domain’, IEEE Trans. Electromagn. Compat., vol. 54, no. 4, Aug. 2012, pp. 826-836

[24] IEC 61000-4-21, Ed. 2.0: 'Electromagnetic compatibility (EMC)' - Part 4-21: 'Testing and measurement techniques - Reverberation chamber test methods', 2011

[25] Pocai M., Dotto I., D’Abreu G., and Festa D.: 'Experimental definition of the lowest usable frequency (LUF) of an aluminum made reverberation chamber with reference to the IEC 61000-4-21 standard', Proc. IEEE Int. Symp. Electromagn. Compat., Portland, OR, USA, Aug. 14-18, 2006, vol. 3, pp. 849-852

[26] Lunden O., Backstrom M.: 'Stirrer efficiency in FOA reverberation chambers. Evaluation of correlation coefficients and chi-squared tests', Proc. IEEE Int. Symp. Electromagn. Compat., Washington, DC, USA, Aug. 21-25, 2000, vol. 1, pp. 11-16

[27] Gifuni A., 'Relation between the shielding effectiveness of an electrically large enclosure and the wall material under uniform and isotropic field conditions', IEEE Trans. on Electromagn. Compat., vol. 55, no. 6, Dec. 2013, pp. 1354-1357

[28] De Leo R., Rozzi T., Svara C., and Zappelli L.: 'Rigorous analysis of the GTEM cell', IEEE Trans. Microwave Theory and Techniques, vol. 39, no. 3, Mar. 1991, pp. 488-500

[29] IEC 61000-4-20, Ed. 2.0: 'Electromagnetic compatibility (EMC)' - Part 4-20: 'Testing and measurement techniques - Emission and immunity testing in transverse electromagnetic (TEM) waveguides', 2010

[30] IEC 61967-2: 'Integrated circuits - Measurement of electromagnetic emissions, $150 \mathrm{kHz}$ to $1 \mathrm{GHz}$ ' Part 2: 'Measurement of radiated emissions - TEM cell and wideband TEM cell method', 2005

[31] Rusiecki A., Aniserowicz K.: 'Evaluation of shielding effectiveness of slotted enclosures by internal stirring', Proc. 20th Int. Conf. on Microwaves, Radar and Wireless Communications MIKON'2014, Gdansk, Jun. 16-18, 2014, pp. 240-243

[32] Rusiecki A., Aniserowicz K.: 'Analysis of differences in results of measurements and calculations of slotted enclosure shielding effectiveness', Proc. 20th Int. Conf. on Microwaves, Radar and Wireless Communications MIKON'2014, Gdansk, Jun. 16-18, 2014, pp. 244-247

[33] Rusiecki A., Aniserowicz K., Duffy A., and Orlandi A.: 'The Feature Selective Validation technique as analysis tool for shielding effectiveness of slotted enclosures', IEEE Trans. on Electromagn. Compat., vol. 57, No. 6, 2015, pp. 1472-1480

[34] 'FEKO user's manual', v. 6.0 (Altair Development S.A. (Pty) Ltd, Technopark, Stellenbosch, South Africa, 2010)

[35] ‘COMSOL multiphysics modeling and simulation software’, v. 4.2, ‘RF module’, (COMSOL Inc., 2011) 
[36] IEEE Standard P1597: 'Standard for validation of computational electromagnetics computer modeling and simulation' - Part 1, 2, 2008

[37] Duffy A. P., Martin A. J. M., Orlandi A., Antonini G., Benson T. M., and Woolfson M. S.: 'Feature Selective Validation (FSV) for validation of computational electromagnetics (CEM). Part I - The FSV method', IEEE Trans. on Electromagn. Compat., vol. 48, no. 3, Aug. 2006, pp. 449-459

[38] Orlandi A., Duffy A. P., Archambeault B., Antonini G., Coleby D. E., and Connor S.: 'Feature Selective Validation (FSV) for validation of computational electromagnetics (CEM). Part II - Assessment of FSV performance', IEEE Trans. on Electromagn. Compat., vol. 48, no. 3, Aug. 2006, pp. 460-467 